\title{
Chiuse poetiche e senso della fine. Spunti per una tipologia
}

\section{Pietro Benzoni}

Università di Padova

\begin{abstract}
Ricordata la sfuggente polisemia del concetto di fine, lo studio (che privilegia l'analisi delle strutture formali, con rilievi di stilistica e metrica) tratta due questioni di carattere generale: quando un testo possa dirsi compiuto e cosa rafforzi una chiusa. Dapprima viene fissata, anche attraverso un'analisi contrastiva, la differenza tra compiutezza e scarto conclusivo, tra effetti di saturazione ed effetti più propriamente clausolari; quindi vengono definite, cercando di valutarne la portata, alcune tra le principali tecniche di intensificazione della chiusa poetica; tecniche sostanzialmente riconducibili a tre ordini di fenomeni: 1) chiuse intensificate da sottolineature tematiche e suggestioni iconiche; 2) chiuse scandite attraverso figure di ricorrenza e variazione; 3) chiuse rilevate da una strategica distribuzione delle informazioni, attraverso dinamiche di attesa e sorpresa, di tensione e soluzione. La varia casistica è illustrata con esempi tratti principalmente, ma non solo, dalla letteratura italiana (e in particolare dalla poesia del Novecento), senza però circoscrivere preliminarmente un corpus omogeneo, coll'intento di mettere in luce come meccanismi conclusivi analoghi ritornino in testi diversissimi per genere ed epoca.
\end{abstract}

Parole chiave: chiuse poetiche, il senso della fine.

\section{Abstract}

Reviewing the ever-fugitive concept of fine, the study (which prioritises the analysis of formal structures, with particular emphasis being given to stylistics and metrics) deals with two questions of a general character: when can a text can be said to be complete, and what elements reinforce a closure? First, also through contrastive analysis, the article sets out the difference between completion and discarded conclusion, between effects of saturation and effects that more properly form part of closure. Thus the author, in an effort to evaluate the scope of this, defines some of the main techniques of the intensification of poetic closure, techniques that are substantially aimed at analysing three types of phenomenon: 1) closures that intensify thematic emphasis and iconic suggestion; 2) closures articulated through figures of recurrence and variation; 3) closures that highlight a strategic distribution of information, through the dynamics of expectation and surprise, of tension and solution. The various types are illustrated with examples taken largely, though not exclusively, from Italian literature (and, in particular, the poetry of the Novecento), without, however, circumscribing an essentially homogenous corpus. The intention is to shed light on the ways in which analogous mechanisms of conclusion recur in the most diverse of texts, throughout different genres and periods.

Key words: poetic closure, the sense of an ending. 
Ora ogni fronda è muta compatto il guscio di oblio perfetto il cerchio.

Vittorio SERENI, chiusa di Solo vera è l'estate...

1.

Prima di entrare nel vivo del discorso, una breve avvertenza. Questo è uno studio sincronico e non diacronico, condotto per affondi ed esemplificazioni, e non con un'indagine a tappeto su un corpus omogeneo: uno studio che vuole, fissate alcune distinzioni teoriche, illustrare tecniche e procedimenti di chiusura, affrontando, sostanzialmente, due questioni: quando un testo possa dirsi compiuto e cosa rafforzi un finale. A tal fine si è scelto di concentrare l'attenzione sulle chiuse dei testi poetici (con esempi tratti principalmente, ma non solo, dalla letteratura italiana) perché, sebbene molti dei meccanismi conclusivi qui individuati non siano solo della poesia, tuttavia le più formalizzate strutture poetiche consentono di coglierli con una certa nitidezza e immediatezza, e, al limite, di ricavare dei modelli descrittivi efficaci; come qui si è voluto suggerire attraverso i frequenti rinvii a tipologie testuali diverse.

Iniziamo dunque tale percorso sottolineando l'intrinseca suggestione del tema, la fine, attraverso le parole di Leopardi:

Tutto ciò che è finito, tutto ciò che è ultimo, desta sempre naturalmente nell'uomo un sentimento di dolore e di melanconia. Nel tempo stesso eccita un sentimento piacevole, e piacevole nel medesimo dolore, e ciò a causa dell'infinità dell'idea che si contiene in queste parole finito, ultimo, ec. (le quali sono di lor natura, e saranno sempre poeticissime, per usuali e volgari che sieno, in qualunque lingua e stile...) 13 dic. 1821 [2251]

Ma se ho richiamato questa nota dello Zibaldone, chiaramente inscrivibile nella leopardiana teoria del piacere, è anche per chiosare il titolo, in cui compare un'espressione, il senso della fine, che - volendo usare un lessico e una distinzione leopardiani - appare vaga, suggestivamente vaga, proprio perché composta non da termini "che definiscono la cosa da tutte le parti», bensì da parole indeterminate, capaci di «destare idee vaste e indefinite», e di suscitare «immagini accessorie». ${ }^{2}$ L'evocatività del sintagma, dunque, scaturisce da una densa polisemia che però ora è opportuno sciogliere. Infatti, da un lato senso consente qui una triplice accezione, il significato, la sensazione, la direzione della fine; dall'altro la fine è un concetto "molle», multiplo e sfuggente che, a sua volta comporta almeno tre distinzioni fondamentali:

1. Si cita, con la numerazione dell'autografo, da Giacomo LeOPARDI, Zibaldone di pensieri, ed. a cura di Giuseppe PACELLA, Milano: Garzanti, 1991, vol. I, p. 1231, corsivi originali.

2. Ibid., vol. I [109-110], p. 123: «Le parole [...] non presentano la sola idea dell'oggetto significato, ma, quando più quando meno, immagini accessorie. Ed è pregio sommo della lingua l'aver di queste parole. Le voci scientifiche presentano la nuda e circoscritta idea di quel tale oggetto, e perciò si chiamano termini perché determinano e definiscono la cosa da tutte le parti». 
I. fine come estremità materiale, come ultima parte, la cui estensione può essere variamente considerata (è la questione del «dove inizia la fine?»), ma che sempre, assieme all'inizio, delimita, de-finisce il testo;

II. fine come esito logico-cronologico di un processo che può essere più o meno coincidente con lo sviluppo del testo; come conclusione che non necessariamente si colloca in posizione terminale, non necessariamente c’è, non necessariamente è una sola;

III. fine come télos, come scopo, come mèta cui un processo tende; quindi come finalismo, come principio diffuso che, quando c'è, può informare il testo più o meno pervasivamente.

Ovviamente queste diverse accezioni di fine sono connesse tra loro, e spesso sono compresenti; ma era importante fissarle subito perchè, in linea di massima, possiamo dire che tanto più esse tenderanno a sovrapporsi e mescolarsi, ossia quanto più, in un testo, la fine materiale coinciderà con un esito logicocronologico, quanto più il finale chiuderà il processo testuale confermandone coerenza e finalismo, tanto più potremo parlare di compiutezza. E, d'altra parte, quanto più il finale si staglierà per contrasto e sarà stilisticamente accentuato e rifinito, tanto più potremo parlare di finale forte. Dico d'altra parte perché, come tra breve cercherò di mostrare attraverso un'analisi contrastiva, un conto è il senso della fine veicolato da una chiusa che, con un effetto di conformità, in qualche modo satura, esaurisce il testo confermandone un qualche principio generativo (ossia un finalismo), un altro è il senso della fine delegato ad una chiusa che, con un effetto di frattura più propriamente clausolare, presenta un imprevedibile scarto conclusivo. Nel primo caso sarà più opportuno parlare di senso del compiuto, nel secondo di senso della chiusura o dello stacco.

Verifichiamo dunque questa distinzione, confrontando Nella mia vita ho già visto le giacche (dalla raccolta Cataletto) di Sanguineti con Tant de sueur humaine (da L'instant fatal) di Queneau:

nella mia vita ho già visto le giacche, $\mathrm{i}$ coleotteri, un inferno stravolto da un Doré, il colera, i colori, il mare, i marmi: e una piazza di Oslo, e il Grand Hôtel des Palmes, le buste, i busti:

ho già visto il settemmezzo, gli anagrammi, gli ettogrammi, i panettoni, i corsari, i casini, i monumenti a Mazzini, i pulcini, i bambini, Ridolini:

ho già visto i fucilati del 3 maggio (ma riprodotti appena in bianco e nero), i torturati di giugno, i massacrati di settembre, gli impiccati di marzo, di dicembre: e il sesso di mio padre e di mia madre: e il vuoto, e il vero, e il verme inerme, e le terme:

ho già visto il neutrino, il neutrone, con il fotone, con l'elettrone (in rappresentazione grafica, schematica): con il pentamerone, con l'esamerone: e il sole, e il sale, e il cancro, e Patty Pravo: e Venere, e la cenere: con il mascarpone (o mascherpone), con il mascherone, con il mozzocannone: e il mascarpio (lat.), a *manuscarpere:

ma adesso che ti ho visto, vita mia, spegnimi gli occhi con due dita, e basta: ${ }^{3}$

3. Cfr. Edoardo SAnguineti, (dicembre 1981) in Segnalibro. Poesie 1951-1981, Milano: Feltrinelli, 1982, p. 345. 


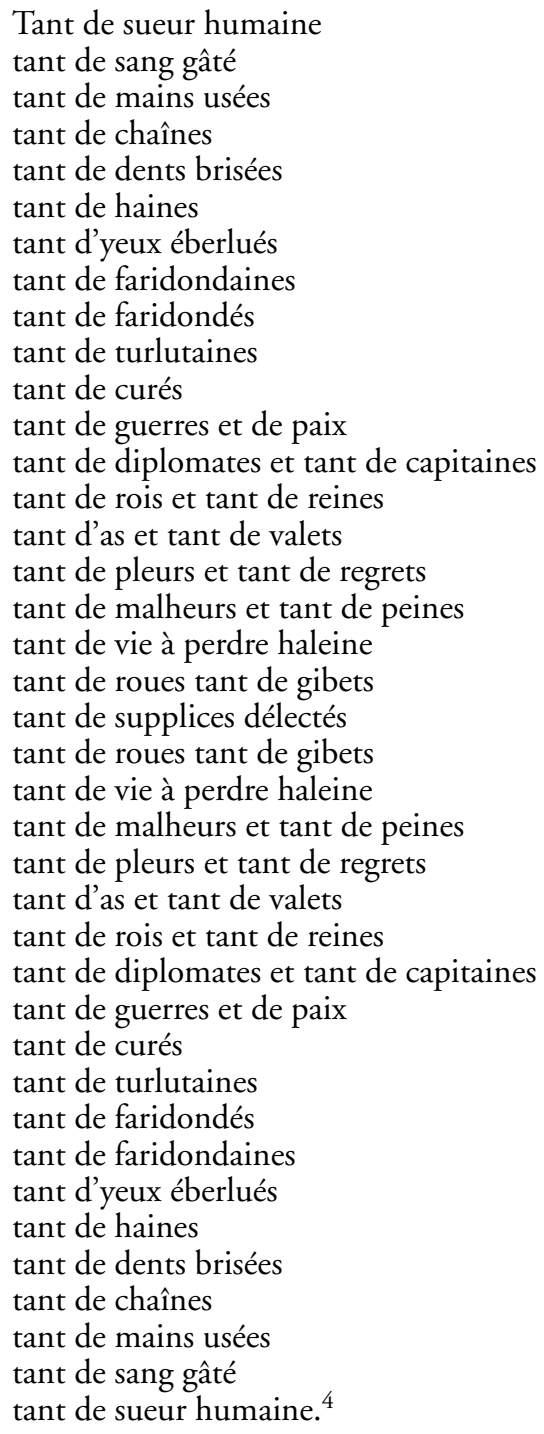

Entrambi i testi si sviluppano attraverso modalità iterative e presentano un uso spinto dell'enumerazione; ma, mentre Sanguineti enumera caoticamente lasciando che siano soprattutto le omofonie ad inanellare gli addendi di una lista teoricamente infinita, Queneau invece costruisce un testo perfettamente speculare, in cui, una volta stabilito il punto di rifrazione (ossia il verso centrale tant de supplices délectés, l'unico verso che non si ripete), il seguito e la 
fine sono predeterminati. Tant de sueur humaine presenta così una struttura alla cui compiutezza ogni parte del testo è necessaria, e in cui ad essere particolarmente rilevato non è il finale (luogo del compimento ma anche della massima prevedibilità), ma il verso centrale, perno attorno al quale ruota il testo. Dove però andrà sottolineato l'intimo contrasto tra la simmetria della struttura e l'incompiutezza logico-grammaticale del discorso: non c'è infatti alcuna frase di senso compiuto, nessun verbo reggente; l'enumerazione dei temi resta sospesa.

Nella mia vita ho già visto le giacche, invece, presenta un finale marcato che, nonostante la punteggiatura anticonclusiva (i due punti finali che «si affacciano» sull'extratesto), riesce comunque a concludere e compattare il componimento, spezzando il meccanismo enumerativo con degli scarti semantici e stilistici significativi.

Ma prima di vedere nel dettaglio quali mi sembra interessante notare come questa strategia conclusiva per certi versi ossimorica (con i due punti finali che, contraddicendo una chiusa altrimenti perentoria, tendono a fare del silenzio post-testuale una continuazione del testo) sia un vero e proprio stilema di Sanguineti. ${ }^{5}$ Il quale, forse a significare l'arbitrarietà di ogni interruzione, spesso costruisce finali marcati da formule e/o tematiche conclusive, ma, nel contempo, «aperti» dai due punti di fine testo. Qualche esempio tratto da Segnalibro. Poesie 1951-1981, cit.: Postkarten 67 «...adesso, che potrei dire / tutto, proprio, non essendo più vivo davvero, non ho più niente da dire, ecco: " che è chiusa del singolo testo e dell'intera raccolta; Purgatorio de l'Inferno 8 «... (noi) dobbiamo morire: "; Purgatorio de l'Inferno 10 «... ma se volti il foglio, Alessandro, non ci vedi niente: "; Purgatorio de l'Inferno 14 «...e dicevo, nel buio, ma immobile: $\neg$ ma non succede niente: »; Postkarten 2 «... buona notte): »; Postkarten 7 «... had a good night: »; Postkarten 50 «vi lascio cinque parole e addio: $\neg$ non ho creduto in niente: »; Postkarten 61 «e allora, caro Sciascia, passo e chiudo: "; etc. ${ }^{6}$

5. Uno stilema di per sé interessante, ma il cui effetto, nell'insieme, mi sembra "annacquato» dall'uso che Sanguineti fa dei due punti, i quali non solo chiudono sistematicamente le sue poesie, ma anche le pervadono e segmentano quasi compulsivamente (un po' come i tre punti la prosa di Céline).

6. E colgo l'occasione da quest'ultimo es., in cui una formula della ricetrasmissione ("passo e chiudo") funge da chiusa poetica, per aprire una piccola digressione. Soprattutto all'interno dei generi, le chiuse, come gli inizi, hanno conosciuto moltissime forme di codificazione e ritualizzazione; e il fatto che ci siano conclusioni stereotipate, formule e modelli tradizionali di finale, fa sì che la fine del testo sia un luogo privilegiato per esporre segnali di riconoscimento e creare effetti di intertestualità. Ora, riprendendo, con esempi nostri, due tipologie fissate da Hamon in un contributo fondamentale — Philippe HAMON, "Clausules», Poétique, n. 24, 1975, p. 495-526 — , la chiusa stereotipata di un dato genere può essere rinnovata pur restando riconoscibile (ad es.: «E vissero infelici e scontenti, né mai ebbero un figlio", "Le sbatto in muso i miei più rancorosi saluti», "La favola non insegna un bel niente»); oppure, come sopra nel caso di "passo e chiudo», una formula conclusiva può essere recuperata, presa in prestito da altri linguaggi, generi o contesti, con effetto più o meno straniante o parodico (ad es.: il «Game over» dei videogiochi, alla fine di una tragedia; "Amen» alla fine di un testo licenzioso; il "Rien ne va plus» del gioco della roulette, alla fine 
Dunque, dicevamo, la chiusa di Nella mia vita ho già visto le giacche (che è anche chiusa dell'intera raccolta Cataletto) si staglia per più di un motivo, o meglio, per più di un effetto di contrasto. Infatti, l'ultimo verso è l'unico che presenta una minima articolazione sintattica e un cambio di soggetto (non più l'io enumerante ma la sua vita invocata), con uno stacco allocutivo che può ricordare quello dei congedi tradizionali. Il tutto sottolineato dalla scansione prosodica; infatti qui (dopo il gradino) sono ravvisabili due endecasillabi canonici: ma adesso che ti ho visto, vita mia (con accenti di $2^{\mathrm{a}} 6^{\mathrm{a}} 8^{\mathrm{a}}$ e $10^{\mathrm{a}}$ ), e spegnimi gli occhi con due dita, e basta $\left(1^{\mathrm{a}} 4^{\mathrm{a}} 8^{\mathrm{a}}\right.$ e $\left.10^{\mathrm{a}}\right)$, entrambi con un secondo emistichio in cui si addensano allitterazioni e assonanze (VÍsTo, VÍTA $m I A$ - Due DiTA, e basTA). Dove però sarebbe falsante parlare di un forte scarto prosodico della chiusa dal momento che, tutto il testo, sotto l'apparente informalità dei versi lunghi, è in realtà contesto di endecasillabi. Semmai quel che va sottolineato è come tale scansione prosodica assecondi il ritmo della sintassi. Infatti qui, nello spazio breve della chiusa, si delinea un tempo di sospensione ( $m a$ adesso che...) e uno di soluzione (spegnimi...) con un procedimento che è uno dei più efficaci e universali per rilevare ritmicamente la chiusa facendone una cellula di senso compiuto. ${ }^{7}$ L'ultimo verso, inoltre, è l'unico che presenta un cambio del tempo e del modo del verbo reggente, con l'imperativo presente spegnimi che interrompe la serie anaforica di passati prossimi (gli ho visto dei versi 3, 5, 8 e 12) su cui è imperniato il testo. Dove andrà notato anche il forte effetto conclusivo del finale con arrivo al presente dell'enunciazione: un meccanismo di chiusura tipicamente narrativo che, soprattutto quando chiude una narrazione al passato, garantisce un effetto di convergenza perché, come ha osservato Genette, «la stessa durata

di un trattato filosofico), ma comunque con efficace sottolineatura della funzione demarcativa. Meno genericamente, nella storia letteraria italiana, un buon esempio di richiamo intertestuale strategico è costituito dalla fortuna del verso clausolare dell' Eneide, «Vitaque cum gemitu fugit indignata sub umbras", richiamato sottilmente nella chiusa dell'Orlando Furioso: «Alle squallide ripe d'Acheronte / [...] Bestemmiando fuggì l'alma sdegnosa, / Che fu sì altiera al mondo e sì orgogliosa» (in corsivo le riprese lessicali più puntuali) e citato, pari pari, con esplicita ironia, alla fine della $1^{\text {a }}$ redazione (1517) del Baldus di Folengo che, appunto, si chiudeva con la morte della strega Pandraga, uccisa però, a differenza di Turno e Rodomonte, non in un duello ortodosso, bensì con un gran calcione. Ossia, in sintesi: la clausola del poema epico è sottesa in chiusa al poema cavalleresco e parodicamente riproposta in clausola a quello maccheronico.

7. Tre ess. volutamente tratti da autori tra loro lontani. 1. Boiardo, chiusa del sonetto proemiale degli Amorum libri: «Ma certo chi nel fior de’ soi primi anni / sanza caldo de amore il tempo passa, / se in vista è vivo, vivo è sanza core», con un verso clausolare che risulta memorabile e lapidario, non solo per via delle varie figure di ripetizione (l'anadiplosi in chiasmo nel centro del verso, l'allitterazione della sillaba $v i$ che ritorna nelle sedi ritmiche-chiave di 2a 6a 8a), ma anche, appunto, per questo effetto di tensione e risoluzione, con protasi e apodosi (significativamente Boiardo riutilizzerà questo verso in chiusa d'ottava, in Orlando innamorato, I, 18, 46). 2. Ciro di Pers, ultimo verso del sonetto Mobile ordigno di dentate rote: «E perché s'apra ognor bussa alla tomba». 3. Montale, chiusa ipoteticamente perentoria del mottetto Il saliscendi bianco e nero.... «Se il chiarore è una tregua, / la tua cara minaccia la consuma». Ma, sui fenomeni di tensione e soluzione sintattica, v. poi il punto 5 . 
della storia diminuisce progressivamente la distanza dal momento della narrazione». ${ }^{8}$ Tutti questi scarti stilistici sono corroborati poi dalla coordinazione avversativa $m a$, dall'uso di un concetto superordinato (vita mia si pone come iperonimo di tutti gli addendi dell'enumerazione) e da suggestioni tematiche conclusive: spegnimento, fine della visione (spegnimi gli occhi) e il performativo basta, proprio nel momento in cui anche il testo sta per finire. Allo stesso tempo, la chiusura di questo testo uniperiodale è ottenuta anche attraverso un effetto cornice, con gli evidenti richiami lessicali (una epanadiplosi con poliptoto) tra l'incipit (nella mia vita ho già visto...) e l'explicit (ma adesso che ti ho visto, vita mia...).

Dunque, tirando le somme del confronto: mentre Sanguineti chiude con un finale marcato, perentorio e performativo, un processo d'accumulo gratuito, Queneau invece porta a compimento lo schema generativo proposto dal testo stesso nel suo dispiegarsi, senza sorprese, con una chiusa prevedibile e quindi, dal punto di vista della teoria dell'informazione, debole.'

\section{2.}

Ma i due principi, saturazione e variazione conclusiva, possono anche coesistere, "cospirare»; come illustra limpidamente la forma perfetta (da perficere "compiere", per e facere "fare fino in fondo") per antonomasia: la sestina lirica.

8. Cfr. Gérard GeneTte, Figure III. Discorso del racconto [1972], Torino: Einaudi, 1976, p. 268; ma v. anche Armine KoTIN MorTimer, La clôture narrative, Mayenne: José Corti, 1985, p. 22-23, e Guy Larroux, Le mot de la fin, Paris: Nathan, 1995, p. 143-145. Quanto agli esempi, si può richiamare la chiusa di Madame Bovary («Il [Homais] vient de recevoir la croix d'honneur") e, più in generale, evidenziare come gli epiloghi narrativi più tradizionali siano spesso caratterizzati non solo da una accelerazione del movimento narrativo, ma anche, appunto, da questo cambio di prospettiva in virtù del quale la narrazione prima condotta al passato, nel finale simula un arrivo al presente.

9. Tant de sueur humaine di Queneau, dunque, è per noi un campione dimostrativo privilegiato del meccanismo speculare, ma, volendo formulare un giudizio di valore, esso ci pare «vittima» della propria prevedibile, claustrofobica esaustività. Più convincenti ci sembrano invece quei testi che configurano strutture speculari compiute, senza però che anche il percorso semantico ne resti imprigionato. Penso, ad es., a Ballata scritta in una clinica (La Bufera) di Montale, Le Memnonidi (Poemi conviviali) di Pascoli e Djinns (Orientales) di Victor Hugo. Nella Ballata scritta in una clinica la specularità riguarda il numero dei versi che compongono ciascuna strofa, dapprima crescente, poi raggiunta la strofa perno, decrescente; così: 1 v. / 2 v. / 3 v. / 4 v. / 5 v. / 6 v. / 7 v. / 6 v. / 5 v. / 4 v. / 3 v. / 2 v. / 1 v. Analogamente, nel poemetto Le Memnonidi, ogni sezione cambia la lunghezza delle sue strofe così: distici - terzine - quartine - strofe pentastiche - quartine - terzine - distici. Ma qui, inoltre, con mirabile effetto di specularità nella specularità, anche il numero delle strofe che compongono ciascuna sezione varia secondo un meccanismo di rifrazioni simmetriche; così: 1 distico introduttivo - 6 distici - 6 terzine -4 quartine -4 strofe pentastiche -4 quartine -6 terzine - 6 distici - 1 distico clausolare. In Djinns le strofe sono tutte ottave e a variare, invece, è la misura del verso; così: 3-4 - 5-6-7 - 8-9-10-9-8 - 7 - 6-5-4 - 3. Quindi, se in Tant de sueur humaine la struttura simmetrica in qualche modo predeterminava forma e contenuto oltre il verso-perno, in questi tre testi invece essa tutt'al più rende prevedibile l'estensione del testo. 
Dove, com'è noto, dapprima vi è un ciclo che può dirsi compiuto alla fine della sesta strofa (se, fermo restando il principio della retrogadatio, ce ne fosse una settima, il suo schema sarebbe identico a quello della prima; così ad es. nella sestina doppia di Petrarca, Mia benigna fortuna e 'l viver lieto, Rvf 332), e quindi vi è un congedo che suggella tale ciclo mutando il gioco della permutazione: stipando, con effetto d'accelerazione riepilogante, le sei parolerima non più in sei ma in tre versi (tre in rima e tre all'interno del verso).

Un'interessante variazione sul meccanismo della sestina è offerto poi dalla petrarchesca S'il dissi mai, ch'i vegna in odio a quella, Rvf206; canzone unissonans che permuta le sue tre rime ogni due stanze secondo il principio della retrogradatio, esaurendo così, nelle sei stanze di cui consta il componimento, il meccanismo combinatorio: lo schema ABBA AcccA delle prime due stanze diviene $\mathrm{BCCB}$ BaaaB nella $\mathrm{III}^{\mathrm{a}}$ e $\mathrm{IV}^{\mathrm{a}}$ e CAAC CbbbC nelle ultime due (segue un congedo con schema $\mathrm{Cbb}\left(\mathrm{a}^{5}\right) \mathrm{C}$ ). Ma, allo stesso tempo, lo sviluppo di questa canzone è scandito da anafore strutturanti (la formula base $S_{i} i$ ' $l$ dissi, amplificata in $S$ 'i' ' l dissi mai all'inizio della $\mathrm{I}^{\mathrm{a}}$ e III $^{\mathrm{a}}$ stanza, apre tutte le prime quattro stanze dove si ripresenta anche ad apertura di piede e di sirma), le cui variazioni più significative (Ma sio nol dissi e I' nol dissi già mai) aprono, rispettivamente, l'ultima e penultima stanza. Ossia, in sintesi, un forte scarto conclusivo in due tempi affianca (e corrobora) il compiersi di uno schema metrico ferreo ed esaustivo.

Ma la sestina è anche un oggetto teorico privilegiato sul cui meccanismo, imprigionante e incantatorio, non a caso, si è soffermato, tra gli altri, uno dei fondatori e teorizzatori dell' OuLiPo, Jacques Roubaud. ${ }^{10}$ Dico non a caso, perché è soprattutto nella letteratura a contraintes, e quindi nella produzione dei membri dell' OuLiPo («la contrainte est à la fois principe de l'écriture du texte, son mécanisme de développement, en même temps que son sens [...] le seul texte admissible, pour la métodhe oulipienne, est celui qui formule la contrainte et, de ce fait même, l'épuise»), ${ }^{11}$ che si possono trovare alcuni dei più ingegnosi esempi di testi compiuti attraverso la saturazione di griglie e schemi prefissati. Si pensi ai sistemi di rotazioni combinatorie che regolano la partitura de Le città invisibili e de Il castello dei destini incrociati di Calvino, ${ }^{12} \mathrm{o}$ a quello che forse è l'esempio-principe, La vie mode d'emploi di Perec, col suo "disegno sterminato e insieme compiuto»(Calvino), su cui ora vale la pena soffermarsi brevemente.

10. Oltre a Jacques Roubaud, «La sextine de Dante et d'Arnaut Daniel», Change, n. 2, 1969, p. 9-38, segnalo Aurelio RonCAGLIA, «L'invenzione della sestina», Metrica, II, 1981, p. 3-41, e Gabriele FrasCA, La furia della sintassi. La sestina in Italia, Napoli: Bibliopolis, 1992 (cui si rinvia per ulteriore bibliografia).

11. Cfr. Jacques RoubAUD, «Les mathématiques dans la méthode de Raymond Queneau», Critique, aprile 1977, p. 402 e 407.

12. Sul «rigoroso e chiuso caleidoscopio di combinazioni finite» de Le città invisibili si veda Pier Vincenzo MENGALDO, «L'arco e le pietre (Calvino, Le città invisibili)», in ID., La tradizione del Novecento. Prima serie, Torino: Bollati Boringhieri, 19962 [1975], p. 433-435; su Il castello dei destini incrociati, Maria CORTI, Il viaggio testuale, Torino: Einaudi, 1978, p. 169-184. 
Ne La vie mode d'emploi, la tensione al compimento è forma e contenuto insieme. L'idea di sfuggire all'arbitrarietà dell'esistenza attraverso regole arbitrariamente scelte ma irriducibilmente perseguite, e quindi l'ossessione del progetto da portare a compimento, il demone dell'esaustività, non sono solo dell'autore; sono anche dei suoi due personaggi più rilevanti: Bartlebooth, e il pittore Valène. Ora, sia l'uno che l'altro muoiono contemplando il fallimento del proprio progetto: Barthlebooth muore davanti al suo puzzle incompiuto e, oltretutto tenendo in mano la tessera sbagliata, Valène accanto alla sua tela praticamente vergine. Ebbene, quel che qui interessa notare è come questo avvenga proprio nelle due ultime estremità del racconto (rispettivamente: fine del $99^{\circ}$ capitolo e fine dell'Epilogo), in due chiuse simili (entrambe fissano fotograficamente, con gelida oggettività, la scena della morte) che si richiamano e rinforzano vicendevolmente con un procedimento che, a grandi linee, può anche essere letto come l'equivalente, nella struttura tematiconarrativa del romanzo, di una delle più archetipiche demarcazioni metriche: la clausola con rima baciata. ${ }^{13}$

La duplice rappresentazione del fallimento, dunque, suggella drammaticamente un testo ultracompiuto. Ultracompiuto sì, nel complesso, ma con una piccola intenzionale lacuna. Infatti nella struttura del romanzo (che, come si sa, è modellata sullo spaccato di un immobile parigino con cento vani ad ognuno dei quali corrisponde un capitolo: una sorta di scacchiera sulla quale l'autore si muove come il cavallo degli scacchi, ricoprendo tutte le caselle senza

13. La clausola con rima baciata (già consigliata da Dante nel De vulgari eloquentia, II, XIII, 7-8: «Pulcerrime tamen se habent ultimorum carminum desinentiae, si cum rithmo in silentio cadunt»: «E tuttavia la disposizione più bella che assumono le terminazioni dei versi finali, è quando scivolano nel silenzio accompagnate dalla rima») definisce alcune delle più tradizionali forme metriche. Ricordo infatti che: nella canzone antica è molto frequente la chiusura della stanza con un distico a rima baciata (la combinatio); nell' ottava narrativa, ad una sequenza di due rime alternate si oppone una terza rima baciata (schema $\mathrm{ABABABCC}$ ); nel sonetto ritornellato, alla consueta struttura del sonetto, viene aggiunto un verso che rima con l'ultimo verso del sonetto stesso, oppure un distico a rima baciata, con rima diversa da quelle impiegate nel sonetto (ad es. Cavalcanti usa lo schema ABBA ABBA CDE DCE FF); nel sonetto caudato generalmente la coda è formata da un settenario in rima con l'ultimo verso del sonetto e da un distico di endecasillabi a rima baciata, con una rima nuova (schema ABBA ABBA CDC DCD dEE); nel sonetto elisabettiano le quartine di rime alternate sono chiuse da un distico con diversa rima baciata (schema ABABCDCDEFEFGG); e, in maniera per certi versi simile, nell'oneginskaya, la strofa di 14 tetrapodie giambiche inventata da Puškin per l'Evgeniy Onegin, un distico con diversa rima baciata chiude una sequenza di versi in cui la quartina viene ricomposta tre volte secondo le diverse possibilità rimiche, rime alterne, baciate, incrociate (schema ab'ab' ccd'd' ef'f'e g'g'; dove l'apice indica la rima maschile); nei melodrammi una rima, per lo più baciata, suole chiudere, prima di un'aria, il recitativo in endecasillabi e settenari sciolti; etc. In linea di principio, naturalmente, la funzione demarcativa della rima baciata sarà tanto più forte quanto più essa si staglierà per contrasto: per intenderci, un conto è la rima baciata che chiude un componimento privo di rime (del tipo ABCDEFGHILM... ZZ), un altro è quella posta alla fine di una successione uniforme di rime baciate (del tipo $\mathrm{AA} \mathrm{BB} \mathrm{CC} \ldots \mathrm{XX}$ ) la cui funzione demarcativa, naturalmente, sarà pressoché nulla. 
mai calcare le stesse ${ }^{14}$ c'è un vano, uno solo, «lo scantinato in basso a sinistra", che, nonostante la mossa del cavallo sia prevista, non viene visitato. Perec lascia cioè il meccanismo sull'orlo del proprio compimento, lo contempla. Con una piccola sprezzatura dell'esaustività che, è stato detto, rappresenta una sorta di clinamen, di errore che dà vitalità al sistema; e che a me sembra possa richiamare da un lato un aforisma di Kraus, «Per essere perfetta le mancava un difetto», dall'altro una riflessione di Cecchi, colpito da un'usanza delle tessitrici Navajo:

Quando una donna Navajo sta per finire uno [dei suoi] tessuti, lascia nella trama e nel disegno una piccola frattura, una menda: «affinché l'anima non le resti prigioniera dentro al lavoro». Questa mi sembra una profonda lezione d'arte: vietarsi, deliberatamente, una perfezione troppo aritmetica e bloccata. ${ }^{15}$

Ma dopo questa digressione su compiutezza e perfezione, concetti che in qualche modo investono l'intera struttura testuale, torniamo ad occuparci delle chiuse poetiche in senso stretto. Con una drastica schematizzazione, che in qualche modo strutturerà il seguito del mio scritto, diciamo che la chiusa può essere intensificata sia con sottolineature tematiche, sia con rifiniture formali quali, in particolare, le figure di ripetizione, sia con una strategica distribuzione delle informazioni, attraverso dinamiche di annuncio e sorpresa, di tensione e soluzione.

\section{Fenomeni di enfasi tematica e di iconicità della chiusa}

$\mathrm{Ci}$ sono dei temi e dei motivi che, se collocati in chiusa, per una sorta di metalinguaggio implicito, sembrano avvalorare la sensazione e l'esperienza della fine, perché ne richiamano, in maniera più o meno evidente, l'idea, il semema. Inevitabile pensare innanzitutto alla morte, evento naturalmente percepito come definitivo e irreversibile. Ma anche al matrimonio, evento convenzionalmente definitivo che, assieme alla morte, è forse la tematica conclusiva universalmente più attestata in narrativa. Forse perché la calma della felicità sancita dal matrimonio (l' "E vissero felici e contenti») e la calma della morte sono, da un punto di vista narratologico, finali-scioglimento equivalenti: entrambi segnano nettamente, l'uno disforicamente, l'altro euforicamente, la fine del conflitto, la fine del divenire, quindi la fine dell'interesse narrativo; come ha detto chiaramente Balzac in Splendori e miserie delle cortigiane: «la felicità non ha storia e gli scrittori di tutto il mondo l'hanno capito

14. Per un approfondimento della ben più complessa meccanica del testo cfr. lo stesso Georges PereC in «Entretien avec Jean-Marie Le Sidaner», L'Arc, n. 76, 1979, p. 3-10 (trad. it. di Elio Grazioli in «Riga», n. 4, Milano: Marcos e Marcos, 1993, p. 90-97) e Odile MARTINEZ, "La vie mode d'emploi: la contrainte come fine», in Monique STREIFF-MORETTI (a cura di), La fine del racconto, Napoli: Ed. scientifiche italiane, 1991, p. 215-233.

15. Cfr. Emilio CeCCHI, Messico [1930], Milano: Adelphi, 1985, p. 51. 
così bene che la frase: "furono felici" termina tutte le storie d'amore»; ${ }^{16}$ e come Tolstoj lascia trasparire collocando nell' incipit di Anna Karenina questa senteziosa affermazione: "Tutte le famiglie felici si assomigliano fra loro, ogni famiglia infelice è infelice a suo modo». ${ }^{17}$

$\mathrm{Ma}$, anche indipendentemente dalla trama e dalla topicità del tema conclusivo, la sensazione della fine nel finale può essere comunicata attraverso sottolineature tematiche più mediate, implicite o allusive. Temi come il silenzio, l'oscurità, il sonno, l'immobilità, l'al di là (la soglia da attraversare), la cancellazione, l'irrigidimento, la sparizione etc., sono tutti riconducibili all'idea della morte e quindi della fine. E innumerevoli poi sono le loro possibili declinazioni e varianti; perché non esistono temi isolati, ma costellazioni, grappoli di temi e motivi. Il procedimento è intuitivo ed è noto ${ }^{18}$ per cui, senza insistere oltre, mi concentro sugli esempi.

Dapprima un es. fittizio per sottolineare come, essendo la chiusa uno spazio variamente circoscrivibile e articolabile, anche parlando di suggestioni tematiche in chiusa, sia importante distinguere i fenomeni di carattere puntuale da quelli protratti e, soprattutto, da quelli dotati di una progressione. Si consideri dunque il motivo potenzialmente «mimetico" della chiusura di un serramento, poniamo una saracinesca: ebbene un conto è chiudere il testo dicendo ...e la saracinesca calò (dove comunque la parola tronca su vocale aggiungerebbe un effetto di clausolarità fonica); un altro è scandire tutto il finale con una descrizione di come la saracinesca cali, svolgendo a poco a poco le sue lamiere ondulate, facendo cigolare vieppiù il rullo, scorrendo sempre più velocemente lungo le guide laterali, sino a quando, in clausola, non urti con fragore metallico il suolo; dunque con progressione ottica, cinetica e acustica che naturalmente intensificherebbe il senso della fine. Ed ora tre ess. letterari.

La chiusa come luogo dell'avvenuta consumazione. La Bougie di Ponge è un componimento che non si limita a descrivere il proprio oggetto bensì tende ad identificarsi con esso (con un'operazione che è l'esatto contrario del «ceci n'est pas une pipe» di Magritte); per cui, come accade anche in altri testi della raccolta Le parti pris des choses, la fine del testo qui viene determinata dalla natura dell'oggetto descritto, fingendo una sorta di necessaria simultaneità (cito l'ultimo capoverso):

[...] Cependant la bougie, par le vacillement des clartès sur le livre au brusque dégagement des fumées originales encourage le lecteur, — puis s'incline sur son assiette et se noie dans son aliment. ${ }^{19}$

16. Cfr. Honoré de BALZAC, Splendori e miserie delle cortigiane [1847], Torino: Einaudi, 1991, p. 75.

17. Cfr. Lev Tolstoj, Anna Karenina [1877], Torino: Einaudi, 1945, p. 1.

18. Si vedano Barbara Herrnstein Smith, Poetic Closure. A Study of How Poems End, Chicago: Chicago U. Press, 1968, p. 172-182; Philippe HAMON, "Clausules», cit., p. 516; Vittorio ColetTI, «Dall'inizio alla fine» [1980], in ID., Italiano d'autore, Genova: Marietti, 1989, p. 150-155; e Marco KunZ, El final de la novela, Madrid: Gredos, 1997, p. 163-186.

19. Cfr. Francis PONGE, Le parti pris des choses, Paris: Gallimard, 1942, p. 16; ibid. un analogo effetto di simultaneità caratterizza le chiuse di Le Pain, "Mais brisons-la: car le pain doit être dans notre bouche moins objet de respect que de consommation», Le Pré: "Voici donc, 
Qui, dunque, la descrizione, reificatasi, si spegne nel finale con il suo oggetto: la candela che affoga nella cera liquefatta, dans son aliment, come si dice in clausola a sottolineare l'inquietante trasformazione del mezzo di sostentamento in strumento di morte.

La chiusa come caduta nel non-testo. Propongo una lettura iconica di due chiuse celebri.

La prima è quella de Le goût du néant di Baudelaire

v. 15 Avalanche, veux-tu m'emporter dans ta chute?

dove l'idea della fine come caduta è avvalorata, da un lato, dal fatto che tale immagine in francese è anche lessicalizzata, ${ }^{20}$ dall'altro, da un effetto iconico specifico del mezzo: infatti, la visione della valanga dalla cui chute il poeta vorrebbe essere trascinato, e quella, di poco precedente, del corpo congelato inghiottito da una neve immensa (v. 11-12: «Et le Temps m'engloutit minute par minute, / Comme la neige immense un corps pris de roideur») sembrano anticipare e amplificare l'imminente «sprofondamento» nel bianco tipografico che generalmente caratterizza la fine del testo nei libri. Bianco che d'altra parte, in quanto non-colore, o colore al margine dello spettro dei colori, non diversamente dal nero, è riconducibile ad una simbologia della fine.

L'altro esempio è dato dalla chiusa perentoria (ma non assoluta: segue il tornar della mente del canto successivo) del quinto canto dell' Inferno dantesco:

Mentre che l'uno spirto questo disse,

l'altro piangëa; sì che di pietade

io venni men così com'io morisse.

v. 142 E caddi come corpo morto cade.

dove, ad acuire il senso della fine, contribuiscono più elementi di natura diversa: la metaconclusività delle tematiche (caduta, allusione alla morte e svenimento di Dante al venir meno del canto), la progressione narrativa che fa di questo svenimento una conclusione quasi fisiologica dell'episodio (la pietà di Dante qui ha raggiunto il suo acme) e le ri-finiture formali con, in particolare, i due poliptoti: morisse...morto a concatenare penultimo e ultimo periodo e, soprattutto, caddi...cade a stringere le due estremità di un verso clausolare sintatticamente autonomo la cui tensione chiusa è corroborata anche dalla fitta

sur ce pré, l'occasion, / Prématurement, d'en finir», e Les Mûres, «Sans beaucoup d'autres qualitées, — mûres, parfaitement elles sont mûres — comme aussi ce poème est fait»; solo che qui è ottenuto altrimenti: con un gioco di parole che collega il piano dell'enunciazione a quello dell'enunciato (cfr. Philippe HamON, "Clausules», cit., p. 516).

20. In francese chute può significare sia "fine inattesa" (ad es. la chute d'une histoire; proverbiali poi le chutes di Maupassant), sia «estremità (ad es. la chute d'un toit «l'orlo di un tetto»), sia "cadenza" (ad es., la chute d'une phrase). Viene in mente poi Spitzer quando, analizzando uno stratificato periodo proustiano, osservava come la chute d'eau clausolare creasse «una splendida chute de la phrase» (cfr. Leo SPITZER, Marcel Proust e altri saggi di letteratura francese moderna, Torino: Einaudi, 1959, p. 236). 
trama di allitterazioni; con una concentrazione di ripetizioni lessicali e foniche che, come ha scritto Spitzer, sembra «rafforzare l'impressione di un'inerzia imposta dalle leggi fisiche». ${ }^{21} \mathrm{E}$ sulla scia di queste notazioni stilistiche sulle figure di ripetizione, passiamo al punto seguente.

\section{Chiuse rilevate e scandite attraverso fenomeni di ricorrenza e variazione}

Cinque sono i meccanismi, basilari, che qui voglio schematicamente fissare e sinteticamente illustrare, cercando di valutarne gli effetti. ${ }^{22}$

I.

Nello spazio breve della chiusa sono ricompattati elementi prima disseminati lungo un porzione di testo più ampia (schema A...B... ...D... ABCD), con un effetto di accelerazione riepilogante o condensazione che naturalmente sarà più $\mathrm{o}$ meno forte a seconda delle modalità di ripresa (bisognerà cioè tener conto di variabili quali l'entità e la natura della porzioni di testo interessate, lo spazio che intercorre tra le occorrenze, la loro successione, etc.).

Tale procedimento riassemblante, che è alla base di alcune delle più codificate forme di chiusura nei generi più diversi (quali la ricapitolazione dei temi trattati nella perorazione classica, le conclusioni riepiloganti di tanta saggistica, il congedo della sestina lirica di cui s'è detto, il finalissimo delle opere musicali in cui tutti gli attori si radunano sulla scena cantando insieme, l'esibizione collettiva al termine degli spettacoli circensi, il gran finale dei fuochi d'artificio in cui conflagrano insieme tutte le figurazioni prima susseguitesi in sequenze distinte, il quinto tempo di un quartetto d'archi, la panoramica finale di tanti film, ${ }^{23}$ etc.), tale procedimento, dicevo, in poesia, è stato descritto sia da Dàmaso Alonso, il quale ha parlato di recolección, sia da Curtius, il quale ha usato la definizione di Summationsschema (schema sommatorio), e ha cercato

21. Cfr. Leo SpITZER, "Il canto XIII dell'Inferno», in ID., Studi italiani, Milano: Vita e Pensiero, 1976 , p. 166.

22. Tali meccanismi, che possono anche coesistere in uno stesso testo, di per sé, sono noti (se non archetipici); il nostro apporto dunque è soprattutto nel taglio e nella sinteticità della sistemazione, nel percorso esemplificativo e in qualche osservazione teorica. Per un approfondimento cfr. comunque il più ampio, ma non sempre convincente (v. poi), Barbara Herrnstein Smith, Poetic Closure, cit., alla voce repetition.

23. Un finale che coniuga le modalità riassemblanti del finalissimo delle opere musicali con quelle tragiche dei film apocalittici, si trova in un film di Altman, Nashville (1975). Qui, infatti, c’è una struttura narrativa ramificata, a montaggio alternato, con molte storie che si sfiorano e raramente si intrecciano, ma che solo nella grande scena finale, destinata a rivelarsi tragica, trovano un punto di convergenza collettivo. Più precisamente, nell'ultima scena, ambientata nel parco cittadino, e dominata da uno scenario che imita il Partenone, ha luogo lo spettacolo a sostegno della campagna elettorale di Hal Philip Walker (ai cui preparativi si era assistito già nella prima scena; quindi con un effetto incorniciante entro la scena ricollezionante): un grande show che richiama, sul palco o tra il pubblico, tutti i principali personaggi del film; tra i quali il giovane Kenny che poi ucciderà con un colpo di pistola la star della canzone Barbara. 
di tratteggiarne la storia individuando in un componimento di Tiberiano la prima attestazione e nell'età barocca il momento di massima fortuna. ${ }^{24}$

E, appunto, per un primo es. canonico, si veda un sonetto di Lope de Vega, che (come evidenziano i corsivi) ricolleziona i temi trattati nelle quartine, addensandoli, ma con diversa sequenza, nel verso clausolare:

El humo que formó cuerpo fingido, que cuando está más denso para en nada; el viento que pasó con fuerza airada y que no pudo ser en red cogido;

el polvo en la región desvanecido de la primera nube dilatada; la sombra que, la forma al cuerpo hurtada, dejó de ser habiéndose partido, son las palabras de mujer. Si viene cualquiera novedad, tanto le asombra, que ni lealtad ni amor ni fe mantiene.

Mudanza ya, que no mujer se nombra, pues, cuando más segura, quien la tiene, tiene humo, polvo, nada, viento y sombra. ${ }^{25}$

$\mathrm{Ne}$ L'Avenir di Apollinaire, invece, la ricollezione investe tutta l'ultima strofa la quale riprende, ordinatamente, elementi sparsi nelle altre strofe del componimento:

Soulevons la paille

Regardons la neige

Écrivons des lettres

Attendons des ordres

Fumons la pipe

En songeant à l'amour

Les gabions sont là

Regardons la rose

La fontaine n'a pas tari

Pas plus que l'or de la paille ne s'est terni

Regardons l'abeille

Et ne songeons pas à l'avenir

Regardons nos mains

Qui sont la neige

la rose et l'abeille

ainsi que l'avenir 26

24. Cfr. Dámaso Alonso, Saggio di metodi e limiti stilistici [1950], Bologna: il Mulino 1965, p. 218-230, e Ernst CURTIUS, Letteratura europea e Medio Evo latino [1948], Firenze: La Nuova Italia, 1992, p. 319-322.

25. Cfr. Lope de Vega, Poesía lírica, Madrid: Espasa-Calpe, 1941, vol. I, p. 188.

26. Si cita da G. Apollinaire, Poesie, Milano: Rizzoli, 1979, p. 220. 
Anche, ne L'ora granda (da Sénere colde) di Biagio Marin, tutta l'ultima strofa è contesta di elementi ricollezionati, ma con variazioni (1 speciera $>17$ specio; 12 ' so ben > 18 el sovo ben) anche vistose (cfr. i v. 13-14 con gli ultimi due):

Xe ferme l'aque che le par speciera: drento le ha 'l siel co' garghe nuvoleta:

là sui àrzini alti fa l'erbeta

che 'l silensio valisa a so maniera.

Lontan, de là de le marine e i dossi, un respirâ del mar solene e largo; un svolo a l'orizonte, a mar, de ciossi e più lontan un bastimento cargo.

El sol va in alto: l'aqua xe un brilante co' foghi virdi e sangue de rubini e svola in sielo l'órdola a scalini per dî $l$ so ben al dolse amor distante.

v. 13 'Desso 'l silensio drento l'aria trema e la zogia fa môve i fili d'erba: adesso la mantina xe superba de la luse che duta la diadema.

E me son l'aqua che fa specio terso e l'órdola che canta el sovo ben, e me son l'aria e son el canto perso che fa tremâ fin l'erba sul teren. ${ }^{27}$

Ancora: come forme di condensazione finale, possono essere considerate le riprese che scandiscono la chiusa del Canto notturno di Leopardi e quella del mottetto Al primo chiaro... di Montale. Il Canto notturno di un pastore errante dell'Asia presenta infatti un'ultima strofa (uno pseudocongedo, come ha evidenziato Blasucci),${ }^{28}$ in cui due vocativi che si erano avvicendati nel corso della lirica, greggia e luna, ritornano in posizione contigua, in punta di verso entro due versi stretti (con un procedimento del tipo II) da anafora e parallelismo sintattico: v. 137-138 «Più felice sarei, dolce mia greggia, / Più felice sarei, candida luna». Il mottetto Al primo chiaro...è invece un testo bipartito in due strofe, i cui rispettivi incipit (Al primo chiaro... v. 8 al primo buio...) sono sinteticamente ripresi nell'attacco del penultimo verso, là dove il componimento, che è formato da un solo periodo, trova anche il proprio compimento

27. Si cita dall'antologia Poeti italiani del Novecento, a c. di Pier Vincenzo Mengaldo, Milano: Mondadori, 1978, cfr. p. 509.

28. Cfr. Luigi BlaSUCCI, «Partizioni e chiusure nelle prime «canzoni libere» (con alcune prospezioni sulle successive)» [1992], in ID., I tempi dei Canti, Torino: Einaudi, 1996, p. 115. 
sintattico (una reggente con sintassi nominale): v. 13-14 «al chiaro e al buio, soste ancora umane / se tu a intrecciarle col tuo refe insisti».

In narrativa, certo, è più difficile imbattersi in schemi sommatori altrettanto nitidi, ma, volendo trovarne qualcuno, credo sia soprattutto negli intrecci di natura teatrale che si debba cercare. ${ }^{29}$ Altrimenti, mi sembra che le forme di riepilogo narrativo più interessanti poggino su moduli più impliciti ed allusivi; come può mostrare il noto passo dell'ultimo capitolo dei Promessi Sposi in cui hanno luogo le nozze tra Renzo e Lucia:

Venne la dispensa, venne l'assolutoria, venne quel benedetto giorno: i due promessi andarono, con sicurezza trionfale, proprio a quella chiesa, dove, proprio per bocca di Don Abbondio, furono sposi. Un altro trionfo, e ben più singolare, fu l'andare a quel palazzotto; e vi lascio pensare che cose dovessero passar loro per la mente, in far quella salita, all'entrare in quella porta; e che discorsi dovessero fare, ognuno secondo il suo naturale. [...] Dopo i due pranzi, fu steso il contratto per mano di un dottore, il quale non fu l'Azzeccagarbugli [cap. XXXVIII, 45-47]

passo magistralmente cadenzato dalle anafore (venne... venne... venne... proprio... proprio... etc.) il cui vertiginoso effetto riepilogante poggia sulle rapide e ammiccanti sottolineature enfatiche (...il quale non fu l'Azzeccagarbugli) con, in particolare, i deittici anaforici (quel benedetto giorno... proprio a quella chiesa... proprio per bocca di Don Abbondio... quel palazzotto... quella salita... quella porta) che nell'insieme, danno vita ad una trama di allusioni capace di rievocare efficacemente antefatti analoghi e diversissimi insieme... insomma la storia.

II.

La chiusa è scandita da figure di ripetizione o da forme di parallelismo, con sottolineatura della funzione clausolare-definitoria, con effetti di memorabilità e sentenziosità. Effetti tanto più forti quanto più la chiusa è autonoma (perché isolata tipograficamente o sintatticamente indipendente), quanto più essa tende a stagliarsi a mo' di epifonema (perché caratterizzata da stilemi della perentorietà quali il presente gnomico, gli avverbi mai, sempre, un lessico asso-

29. Tra i quali propongo di considerare Il giovane Moncada (1972) di Lernet-Holenia, racconto sfociante in una scena corale con tanto di applausi finali (narrativi e metanarrativi insieme): un ironico e caricaturale lieto fine che vede la formazione di più coppie e il tornaconto di tutti. Dove, tra l'altro, si potrà osservare l'emblematicità di una chiusa in cui (a ricordare come il denaro sia motore e fine di tutto il vorticoso intreccio) torna per l'ultima volta un motivo ricorrente lungo tutto il testo: quello dello zampillo di fontana che cadendo sulla superficie dell'acqua risuona come una cascata di pesetas (con un procedimento che può essere considerato l'equivalente in narrativa della chiusa con versorefrain di cui poi al punto v). Oppure si veda come nell'ultimo capitolo di Corazón tan blanco (1992) di Javier Marías siano stipati, in forme raggrumate, alcuni dei motivi prima diffusamente sviluppati. 
lutizzante, etc.), quanto più la sua elaborazione retorica è al di sopra di quella del resto del testo. ${ }^{30}$ Tre esempi brevemente commentati.

Nella chiusa de La quiete dopo la tempesta di Leopardi

v. 50

Umana

prole cara agli eterni! assai felice

se respirar ti lice

d'alcun dolor: beata

se te d'ogni dolor morte risana.

le figure di ripetizione assecondano un effetto di progressione. Più precisamente, l'epigrafe clausolare, beata / se te d'ogni dolor morte risana, risulta ancor più solenne perché si pone come secondo gradino di una climax scandita da ripetizioni sintattiche (felice se... beata se..., con lo stesso soggetto) e lessicali (l'anafora di dolor; il poliptoto ti... te): una sorta di approdo alla assolutezza e perentorietà attraverso l'intensificazione semantica e l'eliminazione di quanto modulava il primo enunciato (assai felice > beata; respirar da alcun > risanar ogni), complice la comparsa del tema, naturalmente definitivo, della morte.

Montale, invece, scandisce la chiusa dell'Arno a Rovezzano (da Satura) con una anafora e una figura etimologica con diffrazione semantica:

v. 17 Altro comfort fa per noi ora, altro sconforto.

E, significativamente, lo fa coniugando un effetto di simmetria con una sua sprezzatura. Infatti, se da un lato l'anafora (Altro... altro) incornicia l'endecasillabo, dall'altro, il forte enjambement (altro / sconforto) spezza la cornice più ampia che cinge tutto l'enunciato, quella tra i sintagmi Altro comfort ... altro / sconforto; il che corrobora iconicamente la desolazione della stretta finale, con la parola sconforto che «resta sola» nel verso più breve di tutto il testo, davvero sull'orlo della fine. Quanto alla figura etimologica, con densa equivocità, il comfort, contrariamente a quanto potrebbe suggerire l'etimo, qui non solo non dà conforto, ma sembra porsi come una causa, un'aggravante dello sconforto. ${ }^{31}$ Come,

30. Chiuse con simili caratteristiche spesso sono accostabili (se non coincidenti; cfr. Paul ZUMTHOR, «L'épiphonème proverbial», Revue de sciences humaines, n. 163, 1976, p. 313-328) a forme concise e sapienziali del ragionamento come gli aforismi e i proverbi; il che ci suggerisce l'idea che un florilegio di chiuse retoricamente calibrate (estrapolabili, sia pure meno agevolmente, anche dai testi in prosa), possa essere una lettura gradevole e interessante (per un modello da evitare, si veda la acritica trascrizione di finali di Roberto SCHWAMENTHAL e Michele STRANiERo, Il Corsaro Nero piange. 365 modi per finire un romanzo, Milano: Baldini e Castoldi, 1996).

31. E vengono in mente le parole che Montale pronunciò ricevendo il Nobel: «...fa impressione il fatto che una sorta di generale millenarismo si accompagni a un sempre più diffuso comfort, il fatto che il benessere (là dove esiste, cioè in limitati spazi della terra) abbia i lividi connotati della disperazione» (Cfr. Eugenio MONTALE, $\grave{E}$ ancora possibile la poesia [1975], in ID. Sulla poesia, a c. di Giorgio ZAMPA, Milano: Mondadori, 1976, p. 8). 
d'altra parte, suggerisce anche la tutt'altro che agevole prosodia: l'endecasilla-

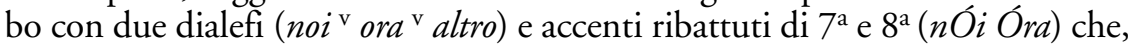
soprattutto se si legge còmfort all'inglese, risulta alquanto accidentato.

Infine, riporto integralmente una poesia di Giudici, Tanto giovane (da $\mathrm{La}$ vita in versi), affinché si possa valutare lo scarto stilistico della chiusa nell'insieme:

«Tanto giovane e tanto puttana»:

ciài la nomina e forse non è

colpa tua - è la maglia di lana

nera e stretta che sparla di te.

E la bocca ride agra:

ma come ti morde il cuore

sa chi t'ha vista magra

farti le trecce per fare l'amore. ${ }^{32}$

Qui, gli unici due versi bimembri, perfettamente bilanciati dalle ripetizioni (a loro volta rilevate prosodicamente), sono il decasillabo dell'incipit, con anafora, e l'endecasillabo dell'explicit, con poliptoto; dove dunque le demarcazioni retoriche della chiusa trovano un corrispettivo in quelle dell'inizio, creando un effetto cornice; il che ci conduce alla seguente tipologia.

\section{III.}

La chiusa presenta una ripetizione che ricollega la fine all'inizio, che incornicia il testo (o una sua parte). La figura retorica cui questo meccanismo può essere ricondotto è l'epanadiplosi (schema A...A); figura che si ritrova in particolare in tutte le ballate con replicazione finale, e che, per definizione, caratterizza una forma tradizionale di poesia per musica come il rondò. Più concretamente si potrebbero vedere, ad es. dall'opera di Di Giacomo, poesie come 'E matina, pe Tuleto, la cui quartina iniziale è identica a quella finale; o la deliziosa $A$ Marechiare le cui strofe pentastiche sono tutte incorniciate da uno stesso verso che però muta di strofa in strofa. ${ }^{33}$

32. Cfr. Giovanni GiudicI, I versi della vita, Mondadori: Milano 2000, p. 28.

33. Il procedimento, d'altra parte, è frequente non solo in poesia, ma anche nella più libere strutture narrative. Si pensi alla complessa strategia incorniciante de La tregua (1963) di Primo Levi, sulla cui soglia iniziale, a mo' di epigrafe, è posta una poesia che poi sarà puntualmente richiamata, chiosata e rimodulata nel brano che chiude il romanzo; o a Lolita (1955) di Nabokov che si chiude come era iniziato: nel nome di Lolita, invocando Lolita; dove cioè la stessa parola, Lolita, a dire anche formalmente l'ossessione dell'io narrante Humbert Humbert, è titolo, incipit ed explicit di tutto il romanzo. Oppure si vedano le chiuse de La certosa di Parma (1839) di Stendhal, di Cuore di tenebra (1902) di Conrad, de Il fu Mattia Pascal (1904) di Pirandello, di Niente di nuovo sul fronte occidentale (1929) di Remarque, de I fiori blu (1965) di Queneau, di Cent'anni di solitudine (1967) di García Márquez, di Se una notte d'inverno un viaggiatore (1979) di Calvino o de Il nome della rosa (1980) di Eco, che richiamano tutte, esplicitamente, il titolo e/o l'incipit. 
Ora però, senza esemplificare, voglio solo sottolineare la natura ambigua degli effetti di circolarità: l'incorniciamento infatti è sicuramente uno dei mezzi più efficaci e tradizionali per con-cludere, per rinserrare il testo, ma, d'altro canto, configurare delle strutture circolari, ad anello, può essere un modo per alludere ad una ciclicità che si rinnova senza fine. ${ }^{34}$

\section{IV.}

La chiusa presenta una variazione che interrompe una serie di ripetizioni invariate. Innumerevoli le possibili realizzazioni, soprattutto se si guarda nell'opera di autori come Pascoli e Di Giacomo, i cui testi sono così spesso imperniati su strutture anaforiche. Si veda ad es. Notte d'inverno di Pascoli, una poesia formata da una collana di madrigali caudati e ritornellanti la cui variazione più significativa si trova appunto in clausola, là dove la chiusa-ritornello dei primi tre madrigali, nell'oscurità, è sostituita da ciò che se ne va:

$$
\begin{aligned}
& \text { [...] } \\
& \text { squillando squillando }
\end{aligned}
$$

v. 11 nell'oscurità.

$$
\begin{aligned}
& {[\ldots]} \\
& \text { tremando tremando }
\end{aligned}
$$

22 nell'oscurità.

$$
\begin{aligned}
& \text { [...] } \\
& \text { gemendo gemendo } \\
& 33 \text { nell'oscurità. } \\
& \text { [...] } \\
& \text { piangendo piangendo }
\end{aligned}
$$

44 ciò che se ne va. ${ }^{35}$

Ma l'effetto conclusivo, ovviamente, sarà più o meno forte a seconda della natura della serie iterativa e delle modalità di differenziazione della chiusa. In particolare si potrà distinguere tra variazioni finali che agiscono per semplice contrasto (del tipo XXX... Y), come quella appena vista, e variazioni finali che in qualche modo sono annunciate o predeterminate dalla serie iterativa $(\mathrm{XXX} \ldots>\mathrm{Y})$, come ad es. nella Guitare di Victor Hugo:

Le vent qui vient à travers la montagne

$72 \quad$ Me rendra fou

34. In particolare, l'epanadiplosi sembra suggerire una ciclicità senza fine quando la ripresa finale si presenta come una autocitazione interna ad un diverso livello di enunciazione, ossia quando vi è anche un effetto di mise en abyme (cfr. Marco KunZ, El final de la novela, cit., cap. III).

35. Cfr. Giovanni Pascoli, Poesie, Milano: Mondadori, 1956, p. 464. 
[...]

Le vent qui vient à travers la montagne

$80 \quad$ Me rendra fou

[...]

Le vent qui vient à travers la montagne

$88 \quad$ Me rendra fou. ${ }^{36}$

Qui infatti, come ha sottolineato Hamon, ${ }^{37}$ la variazione clausolare è avvalorata anche da un più specificico effetto di compimento: quanto reiteratamente annunciato nel distico-refrain (che nell'originale si ripete invariato, non due, ma dieci volte), trova il suo avveramento in clausola. Il tutto corroborato poi da un piccolo ma significativo scarto fonetico: il suono aperto della "a» di rendra si restringe in fine nella vocale in assoluto più chiusa, la «ü» di rendu.

\section{$V$}

La chiusa presenta una ripetizione che non introduce alcuna variazione, bensì conferma la regolarità della serie iterativa. Si osservi ad es., dalle Myricae di Pascoli, Sera festiva: una poesia che presenta (con un hapax metrico nella produzione pascoliana) quattro strofe ognuna formata da una sequenza di sei novenari che è sempre chiusa dal settenario onomatopeico din don dan, din don dan; e questo settenario, a dilatare l'effetto ritornellante, assuona sempre coi due versi precedenti che, a loro volta, si richiamano tra loro, interstroficamente, per l'analogia dei moduli sintattici (cfr. il verso 6 col 20, e il 13 col 27), e per il riecheggiare delle stesse parole-rima; così (cito gli ultimi tre versi di ogni strofa):

[...]

Su gli occhi tu tieni le mani...

Perché? non sai che domani...

v. 7 din don dan, din don dan.

[...]

Tu tieni agli orecchi le mani... tu piangi; ed è festa domani...

14 din don dan, din don dan.

$[\ldots]$

allora perché sonò la campana (perché? non pareva lontana?)

21 din don dan, din don dan. 
[...]

con noi, nella piccola zana:

gridavi; e lassù la campana...

28

din don dan, din don dan. ${ }^{38}$

Se poi si scorre una raccolta come i Canti di Castelvecchio, c'è solo l'imbarazzo della scelta: La partenza del boscaiolo, L'uccellino del freddo, Il brivido, La voce, Per sempre, Il primo cantore, La capinera, La bicicletta, Primo canto, In viaggio sono tutte poesie chiuse e scandite dal ritorno, con regolare frequenza, di un identico verso-refrain (per lo più di carattere onomatopeico).

Questo per una rapida esemplificazione del fenomeno, ma la valutazione del suo effetto è problematica. Barbara Herrnstein Smith ha sostenuto che ripetizioni sistematiche di questo tipo producono un effetto anti-conclusivo, perché l'ultima occorrenza, confermando le precedenti, configurerebbe una regolarità prolungabile ad libitum, per una sorta di forza inerziale. ${ }^{39}$ Ora, che la loro conclusività sia assai minore rispetto a quella delle chiuse con variazioni e contrasto del punto IV, è evidente. Ma vederci unicamente un effetto di strascico e sospensione, "a force for continuation", come fa la Smith, ${ }^{40}$ non è così pacifico. Infatti, da un lato, come ha sottolineato Hamon la ripetizione sistematica potrebbe anche essere interpretata come un principio di prevedibilità, dunque di arresto. ${ }^{41}$ Dall'altro, a me sembra che la forza propulsiva (anticonclusiva) individuata dalla Smith conviva e sia bilanciata da una forza di natura opposta: la forza regressiva insita in ogni ripetizione, che per essere riconosciuta come tale deve richiamare una precedente occorrenza (riconoscere che un $A_{n}$ clausolare è l'ultima occorrenza di una serie regolare $A_{1} A_{2} A_{3}$ $\ldots A_{n}$ implica uno sguardo retrospettivo su tutta la serie, quindi un effetto di chiusura). Senza dimenticare poi l'intrinseca conclusività di un'ultima occorrenza che, ontologicamente, è sempre una variazione: $A_{n}$ è sempre altro da $A_{l}$, e questo a maggior ragione se $A_{n}$ è collocato in clausola (come può suggerire anche l'esperienza più comune: quando le campane suonano le ore, l'ultimo rintocco, anche se di per sé è uguale agli altri, sembra più intenso e protratto solo perché riecheggia nel silenzio).

\section{5.}

La fine, in quanto termine della progressione lineare, indispensabile per cogliere il testo nella sua globalità, è sempre un luogo strategico. Ma tale intrinseca strategicità sarà esaltata da quei testi che, attraverso una determinata distribuzione delle informazioni, fanno della fine il momento di massima intensità, o perché particolarmente atteso, o perché particolarmente sorprendente, o per entrambi i motivi.

38. Cfr. Giovanni Pascoli, Poesie, cit., p. 19.

39. Cfr. Barbara HerrnsteIn SMITH, Poetic Closure, cit. alla voce repetition systematic.

40. Ibid., p. 48 e p. 161.

41. Cfr. Philippe Hamon, «Clausules», cit., p. 508. 
Generalmente si tratta di testi fortemente orientati che possiamo inquadrare entro due poli d'oscillazione: da un lato ci sono i testi continui che configurano un moto progressivo (a climax o anticlimax che sia), e in cui la fine, dunque, tende a porsi come momento culminante, come punto ultimo semanticamente gravido perché gravato da tutto ciò che è venuto prima; dall'altro i testi che avanzano apparentemente divagando o comunque nascondendo il loro effettivo orientamento, per poi riservare alla fine l'agnizione decisiva, il colpo di scena, la rivelazione che rimette tutto in discussione. Il che poi significa distinguere tra una strategicità della fine che agisce soprattutto in forma di proiezione, con effetti di convergenza, e una che invece agisce soprattutto retroattivamente, con effetti risemantizzanti. In entrambi i casi gli ess. sono più facilmente reperibili nei testi narrativi (soprattutto se si guarda, rispettivamente, entro generi come il romanzo di formazione e quello poliziesco), ma i testi poetici mi sembra abbiano il vantaggio dell'immediatezza.

Per i finali-acme, basti ricordare la struttura progressiva della Divina Commedia, con il viaggio ultraterreno di Dante che appunto si conclude, nell'ultimo canto del Paradiso, con la visione di Dio.

Per i finali rivelatori si veda invece, dall'Alcyone di D'Annunzio, L'onda, in cui ad agire in chiusa è un colpo di scena tematico che davvero getta una nuova luce su tutto il testo:

Nella cala tranquilla

scintilla

[...]

il Mare.

Sembra trascolorare.

S'argenta? S'oscura?

A un tratto

come colpo dismaglia

l'arme, la forza

del vento l'intacca.

Non dura.

Nasce l'onda fiacca, sùbito s'ammorza.

[...]

Ma il vento riviene, rincalza, ridonda.

Altra onda s'alza, nel suo nascimento più lene che ventre virginale!

Palpita, sale, si gonfia, s'incurva, s'alluma, propende.

[...]

Il vento la scavezza.

L'onda si spezza, 


\author{
precipita nel cavo \\ del solco sonora; \\ Spumeggia, biancheggia, \\ [...] \\ O sua favella! \\ Sciacqua, sciaborda, \\ scroscia, schiocca, schianta, \\ romba, ride, canta, \\ accorda, discorda, \\ tutte accoglie e fonde \\ le dissonanze acute \\ nelle sue volute \\ profonde \\ [...] \\ E anch'ella [Aretusa] si gode \\ come l'onda, l'asciutta \\ fura, quasi che tutta \\ la freschezza marina \\ a nembo \\ v. 98 entro le giunga! \\ Musa, cantai la lode \\ della mia Strofe Lunga.
}

In questa chiusa rilevata dalla divisione strofica e da uno scarto allocutivo che arieggia la funzione congedo, l'onda tematizzata dal titolo si converte dunque nell' immagine della Strofe Lunga dannunziana. Solo in fine cioè capiamo che, attraverso la lunga e scrosciante descrizione dell'onda - onda che, dapprima incerta nel suo crescere, dopo un apparente fiaccarsi, erompe vittoriosa - la poesia ha cantato, iconicamente e metaforicamente, se stessa; o, meglio, la propria forma; anch'essa fluida, imprevedibile e rid-ondante, ora avvallantesi nei versi più brevi, ora allungata nei settenari (perché anch'essa soggetta ai venti, si vorrebbe aggiungere: in cauda venenum). ${ }^{42}$

Ma se concentrare in chiusa delle impreviste informazioni decisive è un modo per enfatizzare la fine, altrettanto efficace è il procedimento, per certi

42. Simile, ma ben più sottile, il meccanismo di rivelazione tematica utilizzato da Rimbaud ne Le dormeur du val, la cui frase clausolare (sintatticamente autonoma), «Il a deux trous rouges au côté droit», risulta decisiva per reinterpretare e decodificare come metafore o attributi della morte i precedenti predicati; sia quelli isotopi: «Le dormeur [...] un soldat [...] dort $[\ldots]$ il fait un somne $[\ldots]$ dans son lit vert $[\ldots]$ il dort $[\ldots]$ il dort» sia quelli meno marcati: «pâle», «souriant comme un enfant malade», «il a froid», «les parfums ne font pas frissonner sa narine»; lo evidenzia Hamon, il quale sottolinea anche come la rivelazione finale fosse desumibile dalla stereotipia della metafora (il sonno della morte), nonché implicita nel titolo dormeur $=$ dort + meurt (cfr. Philippe HAMON, "Clausules", cit., p. 512). Ma, aggiungiamo noi, si dovrà anche notare come il ritorno (con effetto incorniciante) della parola trou in chiusa conferisca una connotazione meno innocente alla sua prima occorrenza nell'incipit ("C'est un trou de verdure»). 
versi opposto, che fa della chiusa il luogo in cui si risolvono delle attese e delle tensioni protratte lungo il resto del testo. Un procedimento che, nei testi poetici, trova forse la sua più nitida illustrazione nei testi uniperiodali a detonazione finale; ${ }^{43}$ qual è ad es. un sonetto di Petrarca, Gratie ch'a pochi il ciel largo destina..., Rvf 213, i cui primi tredici versi presentano un'enumerazione di temi sospesi, un'accumulatio che solo nell'ultimo verso viene risolta in una frase di senso compiuto (v. 14 «da questi magi transformato fui»); qual è L'anguilla di Montale, poesia che, com'è noto, consta di una sola interrogazione e si articola per apposizioni, con uno schema sintattico (sostantivo + relativa appositiva) che, a creare un effetto di accelerazione, si ripete in forme via via abbreviate, sino a quando, in clausola, compaiono soggetto e verbo reggente («... [l'anguilla] puoi tu / non crederla sorella?»); etc. ${ }^{44}$

In linea di principio ci si può chiedere poi sino a che punto si possa efficacemente caricare un congegno testuale in vista della detonazione finale; perché, mi sembra, l'eccessiva o la troppo complicata dilazione possono anche compromettere l'effetto, un po' come un elastico troppo teso può spezzarsi senza che l'energia accumulata venga indirizzata come si voleva.

$\mathrm{Ma}$, lasciando la questione aperta, mi soffermerò ora invece su un testo breve (in dialetto romagnolo) di Baldini: un caso particolarmente interessante perché la dinamica tensione/scatto qui si avvale delle più disorientanti movenze della lingua parlata a ritardare la comparsa, non del verbo reggente (come avveniva nei due ess. appena visti), bensì dell'oggetto stesso del discorso. Ecco dunque La cambra schéura, un percorso emotivo di accumulo, scoppio e quiete; un grumo narrativo rappreso in tre endecasillabi:

Che pu u m suzéd da rèd, e u $\mathrm{n}$ sint niséun, tla cambra schéura, ad sòtta, tra i pan spórch, a céud la pórta, e a rógg. Dòp a stagh mèi. ${ }^{45}$

Qui, nel giro di soli tre versi, è messa in atto una strategia multipla che allo stesso tempo ritarda e intensifica l'azione liberatoria dell'urlo; che al primo verso è sottintesa (ora come soggetto, ora come complemento oggetto), al secondo è circoscritta da ben tre incisi, al terzo è inquadrata da un'azione preliminare (a céud la pórta), e infine (introdotta da una e con valore non copulativo, ma conclusivo, come rivela l'uso della virgola) è enunciata; insomma anche la sintassi sembra sfogarsi nell' a rógg. Con una convincente mimesi della

43. Di effetti di detonazione finale ha parlato Spitzer studiando il ritmo della frase proustiana (cfr. Marcel Proust, cit., p. 243-246) e poi Renzi analizzando i sonetti uniperiodali di Petrarca (cfr. Lorenzo RENZI, "La sintassi continua. I sonetti d'un solo periodo: C, CCXIII, CCXXIV, CCCLI", Lectura Petrarce, n. 8, 1988, p. 187-220).

44. Solo nell'opera di Montale, i testi uniperiodali con forti effetti di detonazione finale sono almeno altri quattro: Corno inglese, Al primo chiaro..., Accelerato e Luce d'inverno.

45. Cfr. Raffaello BALDINI, Furistìr, Torino: Einaudi, 1988, p. 36. Traduzione (dell'autore): La camera cieca: "Che poi mi succede di rado, e non sente nessuno, / nella camera cieca, di sotto, tra i panni sporchi, / chiudo la porta, e urlo. Dopo sto meglio». 
lingua parlata che però qui, a ben vedere, ha qualcosa di paradossale perché costituisce un estremo artificio letterario: infatti la poca coesione e il cambio di progettualità (ossia l'oscillante finalismo) tipici del parlato sono qui riprodotti con un massimo d'intenzionalità. Dòp a stagh mèi: l'ultimo emistichio, infine, asseconda la calma finale, suggerendo implicitamente l'idea della fine del testo come calo emotivo, come stasi, con un effetto di smorzamento che è tematico e iconico insieme.

A questo punto però bisogna sottolineare come, pur avvalendosi di strategie per certi versi opposte (l'una avendo di mira l'effetto sorprendente, l'altra poggiando su sospensioni e annunci preparatori), le due tipologie sopra esemplificate - il finale rivelatore e quello con soluzione della tensione accumulata - possono coesistere in uno stesso testo. Anche proficuamente; come mostrano i migliori romanzi ad enigma che sanno creare aspettative e tensione per poi, alla fine scioglierle con soluzioni impreviste (ma non incoerenti). ${ }^{46}$ E come ora, più dettagliatamente, si vedrà attraverso l'analisi di La vie c'est comme une dent di Boris Vian (dalla raccolta postuma Je voudrais pas crever), una poesia la cui strategia conclusiva è imperniata su un piccolo, se possiamo chiamarlo così, colpo di scena logico-grammaticale, che «esplode» alla fine di una gradatio, preparato da una certa tensione sintattica; dove, dunque, la chiusa è al tempo stesso apice della progressione e sede di una rivelazione, attesa e sorpendente insieme:

La vie c'est comme une dent.

D'abord on y a pas pensé

On s'est contenté de mâcher

Et puis ça se gâte soudain

Ça vous fait mal, et on y tient

Et on la soigne et les soucis,

Et pour qu'on soit vraiment guéri

Il faut vous l'arracher, la vie. ${ }^{47}$

Il testo dunque si presenta come uno sviluppo graduale, con versi brevi (schema $a^{7} b^{8} b^{8} a^{8} a^{8} c^{8} c^{8} c^{8}$ ) che scandiscono, paratatticamente, l'irreversibile aggravarsi della situazione: dall'assenza di sintomi, alle prime avvisaglie, ai tentativi di cura, sino alla extrema ratio: sino alla necessità di estirpare. Tale progressione semantica, che muove dalla comparazione iniziale «La vie c'est

46. L'imprevisto deve scaturire da una trama coerente (altrimenti la soluzione delude): da questo punto di vista, l'ideale estetico del romanzo ad enigma è assimilabile a quello teorizzato da Aristotele per la tragedia, quando nella Poetica raccomanda che l'intreccio sia annodato in modo che i fatti sopravvengano parà ten doxan "contro ogni aspettativa» e allo stesso tempo, katà to anankaion o katà to eoikos, ossia "concatenandosi attraverso rapporti di necessità o di verosimiglianza».

47. Cfr. Boris VIan, Euvres complètes, Paris: Fayard, 1999, vol. v, p. 234. Traduzione (mia): «La vita è come un dente. / Dapprima non ci si è pensato / Ci si è contentati di masticare / E poi improvvisamente si guasta / Vi fa male, e ci si tiene / E si cercan le cure e che preoccupazioni! / Ma per essere davvero guariti / Bisogna estirparla, la vita». 
comme une dent», viene però sviluppata ambiguamente, giocando sulla intercambiabilità del referente, con pronomi che potrebbero riferirsi tanto a la dent quanto a la vie (in francese, entrambi sono di genere femminile). Tra i due vocaboli, però, è la dent che, prima della fine, si impone all'aspettativa del lettore come tema sottinteso, come soggetto psicologico: ${ }^{48}$ mâcher, faire mal, soigner, arracher, sono infatti tutti verbi che, in senso proprio, presuppongono la concretezza de la dent, piuttosto che un sostantivo astratto come la vie. Solo che, invece, il referente tanto atteso per via della sintassi enfatica (la dislocazione a destra annunciata cataforicamente da ben cinque riprese pronominali), e "ostentato» solo in punta all'ultimo verso, come ultimo rintocco di una triplice rima baciata, è proprio la vie; termine che finisce col chiudere entro una figura ciclica (una epanadiplosi: la vie, è incipit ed explicit) un testo pure così progressivo. Con una rivelazione che non solo ha un fortissimo effetto di retroazione globale, ma è anche stilisticamente enfatizzata da uno scarto metrico-sintattico: nel distico finale si trovano infatti l'unica frase subordinata e l'unico enjambement del testo, ad assecondare il rapido bilanciamento tra un tempo di sospensione (E pour qu'on soit...) e uno di risoluzione (il faut...). Certo, non senza ragione si potrebbe dire che la rivelazione clausolare è già insita nella similitudine iniziale, ma, a guardar bene, si vedrebbe che l'effetto sorprendente del finale è comunque garantito da una sottile incoerenza logicoretorica: quella che era stata presentata come una comparazione, viene invece sviluppata, ma lo capiamo solo in clausola, come una identificazione analogica; basterebbe togliere l'avverbio comparativo comme del primo verso, e l'effetto sorprendente della chiusa sarebbe compromesso.

48. Per soggetto psicologico intendo ciò di cui parla l'enunciato, ciò che l'emittente sembra avere in mente nel momento in cui inizia a dare forma all'enunciato; che non necessariamente coincide col soggetto grammaticale (cfr. Raffaele SimOnE, Fondamenti di linguistica, RomaBari: Laterza, $1998^{9}$ [1990], p. 377 e sg.). 